\title{
UN FECHADO INTRIGANTE
}

\author{
Mario Silveira ${ }^{1}$ y Mónica Patricia Valentini²
}

\section{Resumen}

Durante la excavación del pecio de Zencity (Puerto Madero 2009) se recolectaron un gran número de huesos de distintas especies, entre ellos predomina el de Bos taurus, de los cuales 770 son huesos enteros. El hallazgo de ésta gran cantidad de restos óseos determinó que pensáramos en una serie de hipótesis que explicaran el por qué de ellos en el sitio. Por lo que decidimos realizar un fechado del colágeno en un fémur entero. Esta comunicación intenta explicar con qué nos encontramos ante los resultados de los análisis realizados.
\end{abstract}

Palabras clave: pecio, restos óseos, colágeno, carbono 14

\begin{abstract}
During the excavation of the wreck of Zencity (Puerto Madero 2009) a large number of bones of different species were collected, among them the one of Bos taurus, of which 770 are whole bones. The finding of this large amount of bone remains determined that we would think of a series of hypotheses that would explain why of them in the site. So we decided to perform a closed collagen in a whole femur. This communication attempts to explain what we are faced with the results of the analyzes carried out.
\end{abstract}

Keywords: wreck, bone debris, collagen, carbon 14

\section{Los restos óseos}

En el pecio de Puerto Madero, hallado hacia fines del año 2008 y excavado entre los años 20092010, se hallaron muchos restos, entre ellos óseos, que corresponden a mamíferos, marsupiales, aves, batracios y peces.

\footnotetext{
${ }^{1}$ Dirección de Patrimonio. CABA

${ }^{2}$ Centro de Estudios en Arqueología Subacuática Argentina. Universidad Nacional de Rosario. mopavalentini@gmail. com
} 
Tabla 1.

Detalle de las especies, géneros y cantidades de los huesos analizados. Elaborada por Dr. Mario Silveira.

\begin{tabular}{|c|c|c|c|}
\hline GÉNERO Y ESPECIE & NOMBRE COMÚN & NISP & MNI \\
\hline Bos taurus & Vacuno & 770 & 32 \\
\hline Equus caballus & Caballo & 39 & 3 \\
\hline Blastocerus dichotomus & Ciervo de los pantanos & 3 & 2 \\
\hline Ovis aries & Ovino & 78 & 5 \\
\hline Sus scrofa & Cerdo & 1 & 1 \\
\hline Felis catus & Gato doméstico & 1 & 1 \\
\hline Chaetophractus vellosus & Peludo & 1 & 1 \\
\hline Myocastor coypus & Nutria & 23 & 3 \\
\hline Lutreolina crassicaudtta & Comadreja colorada & 3 & 1 \\
\hline $\begin{array}{l}\text { Lestodelphis albiventris } \\
\text { Tupinambis }\end{array}$ & Comadreja común & 2 & 1 \\
\hline Teguexin & Lagarto overo & 2 & 1 \\
\hline Gallus gallus & Gallinas/pollos & 10 & 3 \\
\hline Meleagris gallopavo & Pavo & 1 & 1 \\
\hline Anas sp. & Patos & 2 & 1 \\
\hline Noithura maculosa & Perdiz chica & 1 & 1 \\
\hline Ciconia maguari & Cigüeña americana & 1 & 1 \\
\hline Myiopssita monachus & Cotorra común & 1 & 1 \\
\hline Lerus dominicus & Gaviota cocinera & 3 & 1 \\
\hline Pterodoras granulosus & Armado común & 61 & 16 \\
\hline Rhindoros dorbigny & Armado tosquero & 33 & 8 \\
\hline Pimelodus clarians & Bagre amarillo & 5 & 1 \\
\hline Pimelodus sp. & Bagre amarillo o blanco & 15 & 3 \\
\hline Salminus brasilensis & Dorado & 10 & 1 \\
\hline Odontesthes bonariensis & Pejerrey & 1 & 1 \\
\hline Prochilodus lineofus & Sábalo & 114 & 5 \\
\hline Lepomus sp. & Boga & 3 & 1 \\
\hline Pseudoplastytoma sp. & Surubí & 10 & \\
\hline Hoplino malabaticus & Tara tira & 1 & 1 \\
\hline Pogonias cromis & Corvina negra & 9 & 1 \\
\hline Catathyridium jenynsil & Lenguado de río & 5 & 1 \\
\hline Hypostomus sp. & Vieja de agua & 37 & 1 \\
\hline TOTAL & & 1.246 & 100 \\
\hline
\end{tabular}

Estos 1.246 huesos, no serían restos de comidas de los tripulantes del pecio, aunque entre ellos reconocimos 2 punzones y un desata nudos (fid en inglés). Este pudo pertenecer a algún tripulante de 
la embarcación u otra que llegara al puerto de Buenos Aires. Salvo este instrumento, pensamos que el resto de los huesos estaban en el ámbito del Río de la Plata y se introdujeron en el casco de la embarcación luego que fuera abandonada por la acción dinámica del río. Estimamos que esto sucedió a fines del siglo XVIII y salvo los huesos de peces y algunas aves acuáticas, el resto de los mismos provienen en realidad de ámbitos terrestres.

El análisis que hemos realizado presenta taxones que nunca se han hallado en excavaciones en sitios históricos de Buenos Aires. Tal el caso de Blastocerus dichotomus, Lutreolina crassicaudtta, Lestodelphis albiventris, Tupinambis teguexi, y aves como Ciconia maguari y los de la familia Ardeidae, Myiopssita monachus y Lerus dominicus. Incluso Myocastor coypus sólo se lo registró en dos sitios: San Juan 338 y Casa Aieta en calle Aieta 1067 (Silveira 2013 y Silveira y Padula en prensa).

La mayor cantidad de huesos corresponde a Bos Taurus $(61,75 \%$ ) con MNI (número mínimo de individuos de 23). En segundo término Ovis aries con 78 fragmentos (6,25\% y un MNI de 5. Los números nos eximen de mayor comentario sobre la dominancia de los restos de vacuno.

Otro punto a destacar es que se hallaron huesos enteros de Bos taurus, un 47 \%. Muchos de ellos correspondientes a los cuartos delanteros y traseros, donde la expectativa de hallazgo, confirmada en la realidad arqueológica, están trozados por el paso de la carnicería y la cocina misma. Incluso se hallaron restos de cornamenta de Bos taurus, un aspecto improbable en sitios de descarte de comidas.

Por cierto que no son restos de comidas de la tripulación del pecio Zencity. En realidad, como dijimos, el casco fue un colector de huesos que por alguna razón estaban en el río, y que por la dinámica fluvial se movilizaban continuamente, hasta que el sedimento cubrió el pecio y atrapó los huesos que hallamos e identificamos. Podemos afirmar que los restos óseos presentaron una dispersión o distribución pareja en todo el sitio y que se encontraron en los niveles de sedimentación natural que comenzaron a cubrir el buque.

Otro hecho a observar y que llama la atención, es la cantidad de restos en general (óseos, cerámica y otros) que contiene el Río de la Plata, por lo menos en el área de hallazgo del pecio de Zencity y que abona la hipótesis de que el proceso de transformación del lugar y de la incorporación de estos objetos en los espacios de la embarcación, tienen que ver con que la misma se transformó en un "imán" de materiales que el agua arrastraba.

\section{Algunas ideas}

Nos queda por cierto, considerar el porqué huesos tan diversos -se han identificado 18 especies aparte de los peces (13 especies)- se hallaban en el Río de la Plata. Se nos presentó como un verdadero interrogante y por ende como un problema de investigación. Esto nos hizo pensar en la posibilidad de un análisis específico que nos brindara más datos para explicar las hipótesis que consideramos en una primera instancia. La verdadera discusión se planteó cuando los resultados no eran los esperados.

Es así que listamos 6 hipótesis que planteamos en nuestras discusiones para explicar esta gran variedad y cantidad de huesos extraídos durante la excavación:

\section{Primera Hipótesis}

Los huesos son producto del descarte de comidas y que fueron arrojados al agua por los pobladores ribereños de los ríos afluentes del Río de la Plata o de barcos que lo surcaban (Silveira 2001). Para esta hipótesis huesos con evidencia antrópica, como huellas de corte y fracturas podrían tener este origen. 


\section{Segunda Hipótesis}

Basura tirada al Río de La Plata por los habitantes de la ciudad de Buenos Aires.

Para sostener esta hipótesis, aparte de huesos con evidencia antrópica (huellas de corte y fracturas), que sin duda son del descarte de comidas, los testimonios históricos informan que desde principios del siglo XVII, en bandos del Cabildo se prohibía tirar basura y animales muertos al río. Uno de ellos nos dice:

...el 31 de enero de 1637, el gobernador Pedro Esteban De Avila ordena a todos los vecinos que "limpien y barran las pertenencias de sus casa, hagan echar la basura en el campo cada sábado de cada semana y tengan la calle limpia y no echen basura en ella..., determinando penas para los infractores ...negra, negro o india que echara basura a la calle... (Prignano, 1998, p.36)

En el siguiente siglo el problema aún seguía vigente

...Un nuevo bando promulgado el 3 de Julio de 1742 insistió sobre el tema al prohibir por enésima vez arrojar animales muertos, basura o desperdicios a orillas de río y en los baldíos. Disponiendo que ello se hiciera en campo abierto y en los alrededores del ejido urbano... (Prignano, 1998, p.43)

Para la misma época otro bando decía, “...Que no arrojen a las calles animales muertos ni carne podrida, lo cual saquen y arrojen al campo, y por ningún pretexto ni motivo a la ribera del río...." (Prignano, 1998, p.47).

Incluso Mansilla en sus Memorias, ya en el siglo XIX, hace referencia a los problemas de la basura (Mansilla, 195, pp.138-139).

En resumen, está claro que los habitantes de Buenos Aires tiraban basura al Río de La Plata, en la que sin duda estaba incluido el descarte de comidas, como asimismo animales muertos y en particular caballos.

\section{Tercera Hipótesis}

De animales que se ahogaron en el Río de la Plata o afluentes, o que murieron cerca de él y fueron arrastrados por la corrientes de estos ríos, en particular en crecientes de los mismos.

La presencia de restos óseos de Bos taurus, Equus caballus y Blastocerus dichotomus, incluso de Ovis aries, pueden atribuirse a este origen. La presencia de huesos enteros de esos taxones pueden deberse al de animales que luego de incorporarse a las corrientes de los ríos se fueron desarticulando. Esto también podría ser válido para huesos de mamíferos pequeños como Lutreolina crassicaudtta, Lestodelphis albiventris, Tupinambis teguexi y de algunas aves, como Ciconia maguari, Lerus dominicus y Syrigna sibilatrix.

\section{Cuarta Hipótesis}

La llamaremos la Hipótesis Darwin y que podría considerarse como una variante de la anterior. Charles Darwin, en su estadía en la provincia de Buenos Aires, observó los efectos de la Gran Sequía que se produjo en la región entre los años 1827 y 1832 en la provincia de Buenos Aires:

...El brazo del río que corre junto a San Pedro estaba tan lleno de cadáveres en putrefacción, que según me dijo el patrón de un barco el hedor lo hacía desde todo punto de vista infranqueable. Indudablemente estos ciento de miles de animales perecieron así en el río. Veíanse sus cuerpos podridos flotar arrastrados por la corriente y muchas, según todas las probabilidades quedaron sepultados en el estuario del Río de la Plata.... (Darwin, 1998, pp.163-164). 
Restos de este origen fueron observados en el río Salado en la provincia de Bs. As. Incluso un análisis de C14 corroboró el hecho cuya antigüedad fue obtenida por datación Radiocarbónica sobre colágeno.

El origen de la tafocenosis fue estimado entre los años 1817 y 1828. Podría tratarse del primer registro de una muerte en masa de ganado pampeano, desarrollada durante el episodio denominado como la "Gran Seca" y vinculado al evento de la Pequeña Edad de Hielo ocurrido durante los siglos XVIII y XIX. (Tonni, et al., 2008, p.307)

Una de las referencias más notables sobre esta sequía fue proporcionada por Charles Darwin quien recorrió la zona durante el año 1833, el período comprendido entre los años 1827 y 1832 se llama la «gran seca», o la gran sequía. Durante ese tiempo fue tan escasa la lluvia caída, que no creció ninguna planta, ni siquiera cardos; los arroyos se secaron, y todo el país tomó el aspecto de un polvoriento camino carretero. Así ocurrió especialmente en la parte septentrional de la provincia de Buenos Aires y meridional de Santa Fe. Murieron un gran número de aves, animales silvestres, ganado vacuno y caballar por falta de alimento y agua. Un hombre me dijo que los ciervos solían meterse en su corral a buscar la poza que se vio obligado a cavar para proveer de agua a su familia y que las perdices apenas tenían fuerza para huir volando cuando se las perseguía. El cálculo más bajo supone que se perdieron sólo en la provincia de Buenos Aires un millón de cabezas.

\section{Quinta Hipótesis}

De animales ahogados y basura arrastrada por el Tercero del Sur cuando se producían lluvias torrenciales. Este desembocaba a la altura de la calle Chile (Lafuente Machain, 1980, pp. 39-40). El análisis de los restos óseos del sitio Imprenta Coni, determinó que parte del material era de redepósitos que provenían del Tercero del Sur, donde se halló caballo (véase en tesis doctoral Mario J. Silveira 2002).

\section{Sexta Hipótesis}

Productos del descarte de los saladeros que se encontraban en ambas márgenes del Riachuelo.

Como antecedentes de estos establecimientos se sabe que los primeros saladeros comerciales aparecen a fines del siglo XVIII, estos toman auge en los primeros años del XIX, en particular con el emprendimiento de la empresa de Rosas, con su establecimiento La Higuerita en el partido de Quilmas (Guiberti 1961:83).

Un testimonio nos dice,

...Según el mismo Pellegrini, desde el año 1816 en ambas márgenes del Riachuelo se establecieron numerosos saladeros. En ellos se faenaba la hacienda vacuna, y se salaban los cueros y la carne, que se exportaban junto con el sebo la grasa los cuernos y hasta los huesos. Se llamaban saladeros a estos rudimentarios establecimientos para distinguirlos de los mataderos, en los que se abatían los animales destinados al consumo de la ciudad. Pellegrini pintó a la acuarela dos saladeros, uno primero en 1830, para el Tableu Pintoresque, poco después de su llegada a Buenos Aires,... (Del Carril y Aguirre Saravia. 1982, p.218)

También en cuanto al operativo en los saladeros tenemos que,

...Otra ventaja del saladero fue la extracción de grasa, que se hacía por medio de tinas con agua hirviendo donde iban a parar huesos, sebo y todas las partes del animal no utilizadas para tasajo. Este anexo absorbería más tarde gran número de ovinos y equinos El animal preferido era el novillo o buey de cinco años de cuero pesado - producto muy valioso- y carne magra, que tomaba 
bien la sal; los vacunos criollos satisfacían a la perfección tales requerimientos.... (Guiberti, 1961, p.91)

Estas prácticas generaban gran cantidad de huesos de Bos turus, e incluso en algún momento de equinos y ovinos. No sabemos bien si los huesos eran exportados, pero la cantidad fue tan grande que muchos debieron ser desechados tirándolos al Riachuelo, que finalmente los conducía al Río de La Plata. La cantidad de huesos enteros bien pueden deberse a esta causa.

La gran cantidad de restos hallados de vacuno (incluidos bueyes) y equino, como la presencia de restos de ovinos hace muy probable que los huesos provengan del saladero. Incluso las huellas de hachuela en algunos huesos pueden deberse al depostamiento.

El cambio del curso del Riachuelo se produjo en marzo de 1783. Un lanchero observo la nueva boca del Riachuelo y lo informó al Cabildo (De Gandía, 1939, p.40). Ya no desembocaba a la altura de la calle Chile, ahora en la actual desembocadura. Esto debió ser el resultado de crecidas del Riachuelo a por temporales de fuertes lluvias. Estas tormentas, que influían en el Río de La Plata, cuya conducta es mas propia de un mar que de un río, debe de haber tenido influencia sobre el pecio hundido, provocando que el sedimento que lo cubría se removiera y permitiera nuevas incorporaciones al pecio. En algún momento es probable que el sedimento tuviera tal espesor que impidiera que quedaran expuestos al agua los restos. Probablemente esto debió ocurrir antes de mediados del siglo XIX, ubicándonos entonces en momentos de pleno funcionamiento de los saladeros sobre el Riachuelo, y por ende el arrojo de huesos al agua del mismo. (Darwin, 1998, p.88)

\section{Consideraciones finales}

Retomando las otras hipótesis, la que llamamos Darwin, como la del saladero, explicaría la alta cantidad de huesos de Bos taurus, en particular muchos de ellos enteros y sin marcas antrópicas. Era oportuno efectuar un fechado de C 14 sobre huesos de este animal.

Se envió a LATYR (La Plata) una tibia entera para fechado. Se obtuvo un fechado "sin valor" (véase al respecto Johnson en Libby 1970, pp.: 170-171)

Se repitió con un fémur entero de Bos taurus.

El análisis realizado en el LATYR (La Plata) con el resultado con fecha 25 de febrero del 2016

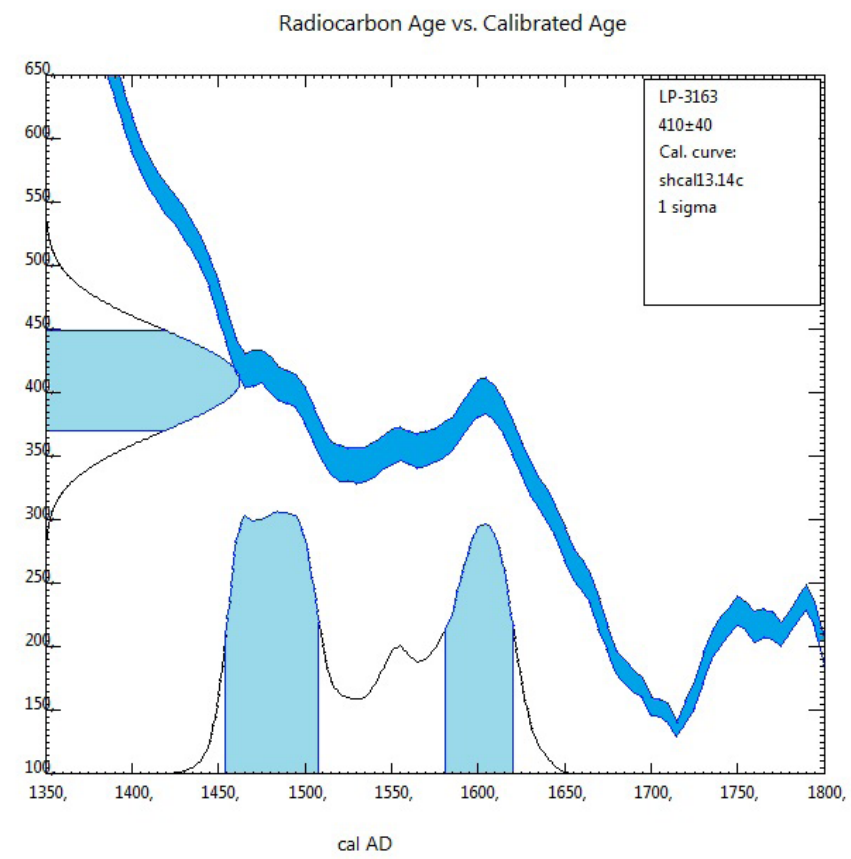

Figura 1: Curva de calibración enviada $\mathrm{x}$ LATYR 


\section{LATYR LABORATORIO DE TRITIO Y RADIOCARBONO \\ Centro de Investigaciones Geológicas (CIG). \\ Facultad de Ciencias Naturales y Museo, UNLP. \\ Consejo Nacional de Investigaciones Científicas y Técnicas, CONICET.}

Sede: Museo de La Plata, Paseo del Bosque s/n, (1900) La Plata, Argentina

Tel: (0221) 427-0648 / Correo electrónico: latynafenym.unjp.edu.ar

Muestra suministrada por: Mónica Valentini

Muestra: hueso. Código: Zen Bos taurus Femur

Sitio: Zencity (Pecio). CABA. Buenos Aires.

Edad Radiocarbónica Convencional: LP- 3163

$410 \pm 40$ años $A P$

Factores de Corrección: $\delta^{13} \mathrm{C}$ ( estimado) : $-20 \pm 2 \%$

Factor multiplicador del error $(\mathrm{K})-1$

Calibración para el Hemisferio Sur : SHCal13. 14c Hogg et al. 2013: Radiocarbon 55 (4)

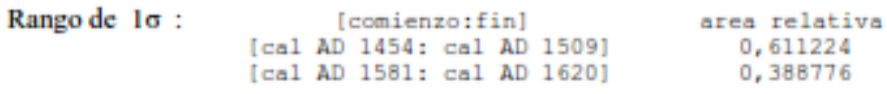

Programa CALIB 7.0.0 Usado en conjunción con Stuiver and Reimer, 1993, Radiocarbon 35 (1).

La Plata: 25 de Febrero de 2016.

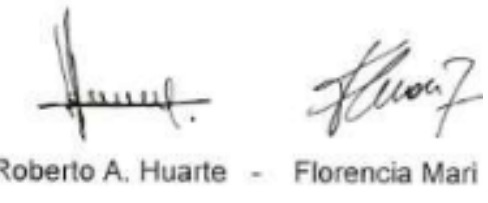

Responsables deI LATYR

\footnotetext{
Calidad analítica del LATYR; participación activa en los siguientes proyectos internacionales:

- Internacional Collaborative Study 1985-1990.

- Quality Assurance Program, IAEA, 1987-1991.

- Third International Radiocarbon Intercomparison, TIRI, 1992-1997.

- Fourth Internacional Radiocarbon Intercomparison, FIRI, 1998-2002.

- Fifth Internacional Radiocarbon Intercomparison, VIRI, 2004-2007.

- Sixth Internacional Radiocarbon Intercomparison, SIRI, 2013.
}

Figura 2: Análisis entregado por el LATYR

Edad radiocarbónica convencional (LP 3163): 410 años \pm 40 años. Es decir ente los años 1646 y 1560. La calibración no altera la fecha.

Este fechado, cercano a la fundación de Garay de Buenos Aires (1580), nos sorprendió y deja varios aspectos de reflexión:

a) en primer lugar la hipótesis Darwin como la de restos del saladero (quinta y sexta hipótesis), 
¿quedarían objetadas? Se puede argumentar que sólo se fecha un hueso, un único animal de hecho, lo que no descartaría la hipótesis.

b) Aunque se trate de un solo animal, explicar su procedencia, para fines del siglo XVI, se hace perentoria. La única explicación que estuvimos analizando es que podría tratarse de los vacunos que trajo Garay cuando fundó Buenos Aires en 1580 (Montoya 1984:73-74). No había ganado vacuno cimarrón en la actual provincia de Buenos Aires para esa época. ¿Estaríamos ante una prueba de la fundación de Buenos Aires?

c) Las demás hipótesis quedan en pie.

Lo recomendable para el caso sería repetir el análisis con por lo menos 10 muestras de otros vacunos. La incógnita de los restos óseos queda sin resolver. Los huesos de Zencity, hasta ahora, nos han otorgado un fechado más que intrigante y han aumentado nuestra incertidumbre...

\section{Referencias}

DARWIN, Ch. (1998). Viaje de un naturalista alrededor del mundo. Bs.As.: Librería Ateneo

DEL CARRIL, B. y AGUIRRE SARAVIA, A. (1952). Iconografía de Buenos Aires. La ciudad de Garay hasta 1852. Buenos Aires: Ed. Emecé.

DE GANDÍA, E. (1939). Historia de la boca del Riachuelo. Ateneo Popular de la Boca. Buenos Aires.

GUIBERTI HORACIO C.E.1961. Historia económica de la ganadería argentina. Buenos Aires: Ed. Solar/Hachette

JOHNSON F. (1970). Reflexiones sobre la importancia de la datación radiocarbónica. En: Libby, W. F. Datación Radiocarbónica. Buenos Aires: Editorial Labor

LAFUENTE MACHAIN, R. (1980). Buenos Aires en el siglo XVIII. Buenos Aires: M.C.B.A.

MONTOYA, A. J. (1984). Como evolucionó la ganadería en la época del Virreinato. Buenos Aires: Ed. Plus Ultra.

PRIGNANO, A. (1998). Crónica de la basura porteña. Del fogón indígena al cinturón ecológico. Buenos Aires: Junta de Estudios de San Jose de Flores.

SILVEIRA, M. (2001). Santa Fe la Vieja. Material subacuático óseo. Análisis y problemas tafonómicos. Arqueología Subacuática. Buenos Aires: Equipo Naya 2001. [CD-ROM].

SILVEIRA, M. (2002). Zooarqueología Histórica Urbana. Ciudad de Buenos Aires. (Tesis doctoral). Recuperado de www..iaa.fadu.uba.ar/cau

SILVEIRA, N. y PADULA, H. Casa Aieta. Ciudad de Buenos Aires. Revista Urbania. En prensa.

TONNI, E. P., BONINI, R. A., MOLINARI, A. E., PREVOSTI, F. J., POMI, L. H., CARBONARI, J. E. y HUARTE, R. (2008). Análisis radiocarbónico en una tafocenosis de la región pampeana (provincia de Buenos Aires, Argentina): Su vinculación con la Gran Seca de 1827-1832 . Intersecciones en Antropología. Tandil, Argentina: Facultad de Ciencias Sociales - UNCPBA.

Recibido: 23/05/2017

Aceptado: 21/09/2017 